\title{
Costs and Benefits of Diagnosing Familial Breast Cancer
}

\author{
Ketil Heimdal $^{\#}$, Lovise Mæhle \\ and Pål Møller \\ Unit of Medical Genetics, Norwegian \\ Radium Hospital, Oslo, Norway
}

\begin{abstract}
Based on results from our surveillance program for women at risk for inherited breast cancer, we have calculated cost per year earned. Norwegian National Insurance Service reimbursement fees were used in the calculations. The calculated costs are based on empirical figures for expanding already established medical genetic departments and diagnostic outpatient clinics to undertake the work described. Cost per year earned was estimated at Euro 753 using our current practice of identifying the highrisk women through a traditional cancer family clinic.

A strategy of identifying the high-risk families through genetic testing of all incident breast and ovarian cancers for founder mutations in BRCA1, will increase the cost to Euro 832. Costs related more to genetic counseling and clinical follow-up than to laboratory procedures. This potential economic limiting factor coincides with a shortage of personnel trained in genetic counseling. The number of relatives counseled to identify one healthy female mutation carrier (i.e. the uptake of genetic testing) is more important to cost-effectiveness than family size. Costs will vary depending upon the penetrance of the mutations detected and the prevalence of founder mutations in the population examined. Prevalences of BRCA1 founder mutations in some high incidence areas of Norway may be sufficiently high to consider population screening. Unlike mutation screening of cancer genes, founder mutation analysis will not identify DNA variants of uncertain clinical significance. Identification of high-risk families through founder mutation analysis of BRCA1 ensures
\end{abstract}

\footnotetext{
\# Correspondence: Dr. Ketil Heimdal, Unit of Medical Genetics, Norwegian Radium Hospital, N-0310 Oslo, Norway, Tel.: +47 229347 68, Fax: +47 229352 19, Email: kheimdal@labmed.uio.no
}

that families with maximum risks are given first access to the limited resources of the high-risk clinics. This may be the greatest contribution to increased cost effectiveness of such a strategy.

The assumptions underlying the calculations are discussed. The conclusion is that inherited breast cancer may be managed effectively for the cost of Euro 750-1,600 per year earned.

KEYWORDS: Familial breast cancer, cost effectiveness analysis, gene testing

\section{INTRODUCTION}

Cancer family clinics have been established all over the world during the last decade. In our clinic, about $80 \%$ of high-risk persons referred belong to breast cancer families. Molecular biological methods are now being introduced. The implementation of genetic testing is potentially very expensive both because of use of elaborate laboratory methods and equipment and because of the labor intensive handling of the information in the counseling situation. Little is known about the costs and benefits of current strategies of breast cancer family programs. We present models that estimate the costs of identifying women at genetically increased risk and following them with intention to diagnose and treat early breast cancer for cure.

\section{MODELS AND ASSUMPTIONS}

All Norwegian inhabitants are insured under the Norwegian National Insurance Service (NIS). This institution reimburses health services such as genetic counselling, clinical examinations in outpatient departments, mammograms, and 
Table 1

Cost in Euro per year of following a Clinical Strategy to identify women at risk and conducting surveillance for hereditary breast cancer. Based on 1998 data from the cancer family clinic of the Norwegian Radium Hospital

\begin{tabular}{lrccr}
\hline & Numbers & Cost per item & Total cost for program & Cost per woman \\
\hline $\begin{array}{l}\text { Genetic counseling } \\
\text { Follow-up }\end{array}$ & $\mathbf{1 , 0 0 0}$ & $\mathbf{1 6 3}$ & $\mathbf{1 6 2 , 8 0 5}^{2}$ & $\mathbf{3 2 6}^{2}$ \\
$\quad$ Clinical examination & $\mathbf{5 0 0}$ & & $\mathbf{1 , 3 8 2 , 2 8 7 ^ { 3 }}$ & \\
$\quad$ Mammography & & 46 & 22,866 & 30,366 \\
$\quad$ FNAC & & 61 & 1,824 & \\
$\quad$ Biopsy & 20 & 91 & 379 & $\mathbf{3 , 0 9 0}$ \\
Total cost of genetic counseling & 5 & 76 & $\mathbf{1 , 5 4 5 , 0 9 2}$ & \\
and follow-up & & & & \\
\hline
\end{tabular}

${ }^{1}$ Based on NIS refunds. ${ }^{2}$ Unpublished data from our activity show that half of genetic counselings lead to enrolment of the woman in the surveillance program. ${ }^{3}$ Based on following the woman 25 years (from 35-60 years of age). Cost of follow-up one year is Euro 55,291. ${ }^{4}$ According to observations that fine needle aspiration is done at $4 \%$ and open biopsy at $1 \%$ of examinations [5].

Table 2

Comparison of cost effectiveness

\begin{tabular}{lcc}
\hline & Cancer family clinic strategy & Founder mutation strategy \\
\hline Cost to enroll one woman in the surveillance program & $326^{1}$ & $6,596^{2}$ \\
Cost of surveillance (table 1) & 2,764 & 2,764 \\
Total cost per woman & $\mathbf{3 , 0 9 0}$ & $\mathbf{9 , 3 6 0}$ \\
Cost per cancer detected & $16,933^{3}$ & $18,720^{4}$ \\
Cost per year saved & $753^{5}$ & $832^{4}$ \\
\hline
\end{tabular}

${ }^{1}$ Cost of genetic counseling (Table 1 ). ${ }^{2}$ Cost to identify one healthy female mutation carrier is computed from table 3 (26 families, 15 healthy members per family of whom $1 / 6$ are healthy female mutation carriers). ${ }^{3}$ Assuming detection of breast cancer in 0.073 of the women per year over 25 years, i.e. $18.25 \%$ breast cancer during surveillance. ${ }^{4}$ Assuming detection of breast cancer in 0.02 of BRCA1 mutation carriers per year over 25 years, i.e. $50 \%$ breast cancer during surveillance. ${ }^{5} 75 \%$ cure rate and 30 years earned per woman cured, see text.

molecular diagnostics. The NIS rates were used in all calculations described. In addition, patients pay Euro 20 per clinical examination and Euro 13 for mammography. These costs are included in the calculations.

\section{Current practice}

We first calculated costs based on our current cancer family clinic (Table 1). About one in two of the individuals receiving genetic counseling for breast cancer risk in 1998 were enrolled in the follow-up program. The cost of enrolling one woman in the high-risk program therefore was set to twice the cost of genetic counseling. The women were offered annual clinical examination and mammography. Four per cent in addition needed fine needle aspiration (FNAC) and/or biopsy [5]. Generally, the women are enrolled at age 35 and continue in the high-risk program until age 60 after which they continue with mammography screening alone. From these data, the cost of following one woman through the period in which she needs special intervention can be calculated. We have previously published annual incidence rates of $0.73 \%$ for breast cancer in our series [7], i.e. $18.25 \%$ invasive breast cancer in 25 years of surveillance. The cost of diagnosing one breast cancer case may be calculated as the cost of enrolling one woman in the program and follow-up costs to approximately Euro 16,900 ${ }^{1}$ (Table 2).

Prolonged life (years earned) was used to measure benefit. The median age of breast cancer diagnosis in the families is in the late forties. Life expectancy in healthy Norwegian women of age 50 is about 80 years. Untreated

\footnotetext{
${ }^{1}$ All calculations use rates as of May $18^{\text {th }} 1999: 1$ Euro $=$
} NOK $8.20=1.07$ US dollars 
breast cancer was assumed to be invariably fatal. Current Biomed2 series indicate $89 \%$ five-year survival in familial breast cancer prospectively demonstrated at an average age of 48 years [6]. From these data we estimate a $75 \%$ cure rate and 30 extra years of life per woman cured. The cost of one year earned may then be taken as Euro 753 (Table 2).

\section{Molecular testing for founder mutations in BRCA1}

We next estimated the costs for testing all breast and ovarian cancer patients in one year in Norway for frequent BRCA1 mutations (Table 3). The result was used as a starting point to estimate the number of families with BRCA1 related disease and to calculate total costs for running the program for one year and costeffectiveness of a founder mutation detection strategy to identify women in families at risk for hereditary breast cancer. In Norway, inherited breast ovarian cancer families are often seen and we have previously shown this to be due to founder mutations in BRCA1 [2]. Two of these mutations have been shown to account for $3 \%$ of all ovarian cancer in a consecutive series [1]. Breast cancer is five times as frequent as ovarian cancer in Norway. Assuming these mutations to give the same number of breast cancers as ovarian cancers (unpublished), i.e. assuming a frequency of these mutations of $0.6 \%$ in breast cancer, cost of determining carrier status in women affected with breast and/or ovarian cancer may be calculated. The calculation is based on current practice, which is to extract DNA and run direct tests for these two mutations. All positives in newly identified families are then verified by direct sequencing of both DNA strands. It is seen that the annual laboratory cost of identifying the families is less than Euro 235,000.

According to Norwegian law, healthy subjects must receive genetic counseling in conjunction with genetic testing. Women affected with cancer are not subjected to counseling by geneticist prior to testing. They are offered testing by their physician. The costs of clinical handling of the proband are therefore not included in our models. Once a mutation is found, the patient is referred to a geneticist for counseling. For the healthy relatives seeking testing, current practice includes two counseling sessions: one pre-test and one post-test to discuss results. In Table 3, we have calculated costs assuming 15 healthy relatives per family to appear for testing. In our experience, $1 / 3$ of the counselees are mutation positive so we assumed that $1 / 6$ were mutation positive women in the relevant age group (35-60 years).

Cost-effectiveness for the founder mutation model is presented in Table 2. Currently,

Table 3

Estimated cost per year following a Founder mutation detection Strategy to identify women at risk for hereditary breast cancer. Based on examining all Norwegian patients with breast or ovarian cancer detected during one year

\begin{tabular}{|c|c|c|c|c|}
\hline & Numbers & Cost per item & Sums & Totals \\
\hline \multicolumn{5}{|l|}{ A. Molecular biology costs } \\
\hline \multicolumn{5}{|l|}{ Identification of families with founder mutation } \\
\hline DNA extraction & $2,593^{1}$ & 24 & 61,347 & \multirow{5}{*}{$\mathbf{2 3 4 , 8 7 9}$} \\
\hline PCR based quick test & 2,593 & 65 & 167,596 & \\
\hline Confirmation of mutation by sequencing & $26 * 2$ & 114 & 5,936 & \\
\hline Testing of 15 family members per family & $390 * 2^{2}$ & 80 & 62,590 & \\
\hline \multicolumn{4}{|l|}{ B. Clinical genetic costs } & \\
\hline Genetic counseling of proband & 26 & 163 & 4,233 & \multirow[b]{2}{*}{131,221} \\
\hline Genetic counseling pre/post testing & $390 * 2$ & 163 & 126,998 & \\
\hline $\begin{array}{l}\text { C. Total cost to identify } 26 \text { families with BRCA1 mutation, } \\
\text { counsel and test healthy relatives }\end{array}$ & & & & 428,690 \\
\hline
\end{tabular}

${ }^{1}$ Based on 439 ovarian cancer cases and 2,154 breast cancer cases per year (Norwegian Cancer Registry, 1995). ${ }^{2}$ All predictive tests are repeated once for confirmation of result in an independent sample. 
mutation carriers receive the same follow-up as other at-risk women. Therefore, the cost of following these may be taken from Table 1. Penetrance for at-risk persons is higher than for women identified by family history alone. The extra laboratory cost to identify mutation carriers was mostly outweighed by this so that the cost per year saved was similar to that of the cancer family clinic strategy detailed above.

\section{Impact of varying the assumptions of the models}

\section{Efficacy of intervention}

The prognosis of BRCA1-related disease with follow-up influences cost-effectiveness of the founder mutation strategy. If none of the women are cured, the strategy is not cost effective. If the cure rate is only $50 \%$, cost per year earned is estimated to increase by $50 \%$ to Euro 248 .

If BRCA1 related disease has a much worse prognosis than other hereditary breast cancer, then the combined European data on follow-up show that BRCA2 (or BRCA-X) related breast cancer must have a much better prognosis. In that case, testing for frequent BRCA2 (BRCA-X) mutations (if found), will be highly costeffective. An alternative strategy then would be to test for BRCA1 mutations, advise prophylactic surgery for mutation carriers, and implement surveillance in the rest of the families. We do not have data to estimate cost-effectiveness for such strategies.

\section{Uptake of testing and family size}

Costs to identify women at risk to be enrolled in screening programs from the cancer family clinic model depend on the fraction of counselees so identified. However, within reasonable limits, this fraction is not critical since the main part of the cost derives from the follow-up program (Table 1).

Since genetic counseling is a major part of the costs in the detection by the founder mutation model, number of patients counseled per healthy female mutation carrier will influence the cost effectiveness of this strategy. The fraction of counselees being female mutation carriers is influenced by uptake of testing and by who is accepted for counseling. The presented model assumes $100 \%$ uptake of genetic testing among counseled relatives. In our cancer family clinic, we have $80 \%$ uptake of testing [8]. Incorporating this into the model increases cost by $10 \%$ to Euro 918. Other centers have reported lower uptakes of testing [3,4]. This may increase costs substantially. For example, cost would increase by $68 \%$ to Euro 1401 if uptake of testing is $40 \%$. The most cost-effective strategy is, of course, to accept for counseling only females in the target group for the mutation analysis. This would also solve the expected manpower shortage. However, we find such a putative practice to be outside current Norwegian legislation and ethically unacceptable.

The size of the family has relatively little impact on cost-effectiveness as demonstrated by the example of reducing the number of healthy relatives requiring testing from 15 to 10 persons. This increases cost less than 20\% from Euro 832 to Euro 995.

\section{Intensified screening programs}

The main effect on costs from the screening program is in the number of screening examinations, while increasing the fraction of FNACs and biopsies have minor impact (Table 1). BRCA1 related breast cancers may have higher growth rates that other breast cancers possibly justifying more frequent examinations in surveillance protocols for mutation carriers. Doubling the number of screening examinations for BRCA1 mutation carriers would increase cost per year earned by $30 \%$ in the presented models, to approximately Euro 1078.

\section{Penetrance of founder mutations}

The penetrance of BRCA1 mutations has great impact on the cost-effectiveness of the programs. Halving the penetrance to $1 \%$ per year, doubles the cost per year earned. These estimates probably represent a worst case analysis.

\section{Prevalence of founder mutations}

Total costs to run the program and cost effectiveness of the founder mutation model is 
dependent upon the prevalence of founder mutations. There will be some reductions in cost per year earned with increased prevalence from the $0.6 \%$ demonstrated in Norway within reasonable limits e.g. up to $2 \%$ (equivalent to $10 \%$ of ovarian cancers). The latter mutation detection rate gives a figure of Euro 611 per year earned and is not unreasonable if there are other frequent mutations in Norway. Adding detection of more founder mutations to the initial mutation screen can easily be done. The model predicts $10 \%$ increase in cost to detect one cancer assuming no increase in the total incidence of founder mutations for adding three primer pairs to the initial screen. However, since an increased prevalence would mean identification of an increased number of families and healthy relatives to be counseled, the total cost of the program would increase dramatically. It can be estimated at Euro 700,000 per year (plus the costs of surveillance of mutation carriers) to run the program on a national scale for $2 \%$ prevalence. More seriously, the number of genetic counseling sessions, would increase dramatically, beyond the capacity of the medical genetic departments. This emphasizes that such a program cannot be implemented unless there is an increase in counseling capacity and/or a change in current counseling practices.

Conversely, the cost per year earned will grow exponentially with diminishing mutation prevalence. The population prevalence of founder mutations may well be in the $0.1-0.5 \%$ range in the southwestern parts of Norway. Using five founder mutations in the initial screen, costs would be in the order of Euro 9,800-2,400 per year earned for population screening in such areas. The total cost of running the program as population screening with mutation prevalence in these ranges would be dominated by the laboratory costs since comparatively few families needing genetic counseling would be found. It is a prerequisite to population screening that the screened women be informed about the program by means other than genetic counseling because of the prohibitive costs and manpower requirements of the latter. From a cost-benefit point of view, population screening in parts of Norway may be advocated. Of course, population screening involves other considerations outside the scope of this paper.

\section{Costs if laboratory and clinical fees are increased}

We have used NIS refunds to the health service to calculate costs. In Norway, these are realistic to cover all expenses for expanding already established services. Establishing new services will increase costs. The figures on costeffectiveness presented by different groups will necessarily differ because of the methodology used. Reported figures mainly reflect local traditions and costs involved in establishing and running a health service. On the clinical side, these costs vary widely while laboratory costs are more standardized between countries. In our clinic in 1998, the NIS refunds covered $1 / 3$ of our total costs, while each additional genetic counselor earned his/her salary by NIS refunds. If the cost of clinical genetic management is trebled and the laboratory cost doubled, the cost of an extra year earned will still be in the order of Euro 1598 (founder mutation strategy) to 110 (clinical strategy).

\section{Screening for unknown mutations}

Current technology for comprehensive mutation screening of whole genes is expensive (Euro 2250 for both BRCA1 and 2). To date, research laboratories have identified such "private" mutations without charge in a number of families. Following disease-associated variants in families involves the same costs as following families with founder mutations. Use of a battery of current mutation screening technologies including direct sequencing will uncover DNA variants of uncertain clinical significance. Determining the clinical significance of these variants involves extensive use of clinical medical expertise and is therefore limited by the availability of such expertise - and very costly. However, these potential problems do not need to be addressed at this time. The founder mutation detection strategy considered in this paper will not produce such unclear results. The clinical capacity of cancer family clinics serving 
populations with strong founder mutations will be started by the families identified with founder mutations and private mutations identified in research settings for the next years to come. By the time these families are properly dealt with, it is expected that cheaper and better DNA technology will be available. PTT as a screening tool may find nothing but frame-shift mutations with high penetrance.

\section{Costs and benefits not considered}

Psycho-social consequences of cancer family clinics may be considered costs. Adverse effects of current activities have so far not been detected [8]. In contrast, our experience is that lack of availability of health care in this group of patients may initiate adverse psycho-social reactions in the families. These questions are currently being evaluated.

Both strategies discussed aim at curing, not preventing breast cancer. Women with incurable breast cancers need long term treatment, nursing, and care. Furthermore, these women (and for shorter periods of time, their relatives) will be out of work and also have the right to sick leave and disability pensions. Avoidance of such costs may be included in cost-benefit calculations as benefits. It may be possible to calculate how many patients cured may be needed to balance the total cost of running the cancer family clinic. The data needed are not available to us, and such calculations are outside the scope of the present paper.

Costs related to establishing cancer family clinics and laboratories including costs to train the personnel needed have not been considered. Laboratories are in the process of becoming automated. In contrast, these costs may be substantial on the clinical side.

\section{DISCUSSION}

Under the assumptions given, the (founder) mutation testing approaches have similar costeffectiveness. Under the most likely assumptions we find that it is slightly more cost-effective to identify the families by the traditional cancer family clinic approach. The highest costs of these programs lie on the clinical side (genetic counseling and clinical follow-up), and not within the laboratory. The more effective a direct search for mutations becomes, the larger part of the total cost will be on the clinical side. Heavy reliance on screening for unknown BRCA1/2 mutation should not considered at this point since it is expensive, expected to become automated soon and therefore much cheaper, and because it in some instances produces unclear results. The potential limiting economic factor on the clinical genetic side coincides with a manpower shortage in genetic counseling, at least in Norway. In this possibly lies one of the major differences in costeffectiveness between the clinical and the founder mutation detection strategy. Identification of high-risk families through founder mutation analysis of BRCA1 ensures that families with maximum risks are given first access to the limited resources of the high-risk clinics. With the notion that BRCA1 related cancer has similar prognosis to other hereditary breast cancer, this may be the greatest contribution to increased cost-effectiveness of this strategy. Current data are insufficient to evaluate this and continued monitoring of highrisk families is mandatory.

An increased laboratory capacity for detecting known mutations may be established rapidly. Training of personnel for genetic counseling takes time. Decisions today on the education of more genetic counselors will determine how these families are handled in the years to come.

In BRCA1 mutation carriers, there is also an increased risk of ovarian cancer. There is a lack of data on the efficacy of ovarian cancer intervention. It is not possible to calculate the cost of the total program until such data are available. However, oophorectomy after childbearing age may avoid more than $75 \%$ of familial ovarian cancers [9]. Assuming such treatment, our models may be valid for breast ovarian cancer families as well since the genetic costs are the same and cost of prophylactic oophorectomy will not substantially change the results.

The most important assumptions of the model 
to consider are the extent and efficacy of the surveillance program, the fraction of counseled relatives that are female mutation carriers/the total number of relatives accepted for counseling, and the penetrance of the mutations detected. However, with the important exception that the prognosis of BRCA1 related cancers is unclear, variation of assumptions within limits as expected from our clinical practice, leads to the conclusion that inherited breast cancer may be cured for the cost of Euro 750-1,600 per year earned.

\section{References}

[1] Dørum, A., Hovig, E., Trope, C., Inganas M. and Møller, P. 3\% of Norwegian Ovarian Cancers are caused by BRCA1 1675 delA or 1135insA. Eur. J. Cancer 35, (1999) 779-781.

[2] Dørum, A., Møller, P., Kamsteeg, E.J. et al. A BRCA1 founder mutation, identified with haplotype analysis, allowing genotype/phenotype determination and predictive testing. Eur. J. Cancer [A] 33A, (1997) 2390-2392.

[3] Lerman, C., Narod, S., Schulman, K. et al., BRCA1 testing in families with hereditary breastovarian cancer - A prospective study of patient decision making and outcomes. JAMA 275, (1996) 1885-1892.
[4] Lerman, C., Schwartz, M.D., Lin, T.H., Hughes, C., Narod, S. and Lynch, H.T. The influence of psychological distress on use of genetic testing for cancer risk. J. Consult Clin. Psychol. 65, (1997) 414-420.

[5] Møller, P., Evans, G., Andersson, E., Mæhle, L., Lalloo, F., Heimdal, K., Steel, C.M. and the Biomed2 Demonstration Programme on Inherited Breast Cancer. Use of cytology to diagnose inherited breast cancer. Disease Markers 15, (1999) 206.

[6] Møller, P., Evans, G., Haites, N., Steel, C.M., Reis, M.M., Vasen, H., Gregory, H., Hodgson, S., Apold, J., Lalloo, F., Mæhle, L., Andersson, E., Heimdal, K. and the Biomed2 Demonstration Programme on Inherited Breast Cancer. Effectiveness of early diagnosis of inherited breast cancer. Disease Markers 15, (1999) 179186.

[7] Møller, P., Maehle, L., Heimdal, K., et al. Prospective findings in breast cancer kindreds: annual incidence rates according to age, stage at diagnosis, mean sojourn time, and incidence rates for contralateral cancer. Breast 7, (1998) 55-59.

[8] Reichelt, J.C., Heimdal, K., Dahl, A.A. and Møller, P. Uptake to BRCA1 genetic testing, anxiety and depression in Norway. Disease Markers 15, (1999) 139-143.

[9] Stratton, J.F., Gayther, S.A., Russell, P. et al. Contribution of BRCA1 mutations to ovarian cancer. N. Engl. J. Med. 336, (1997) 1125-1130. 


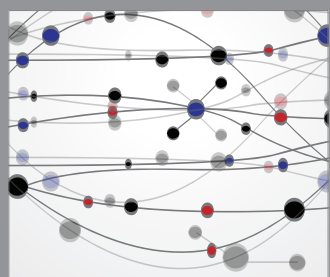

The Scientific World Journal
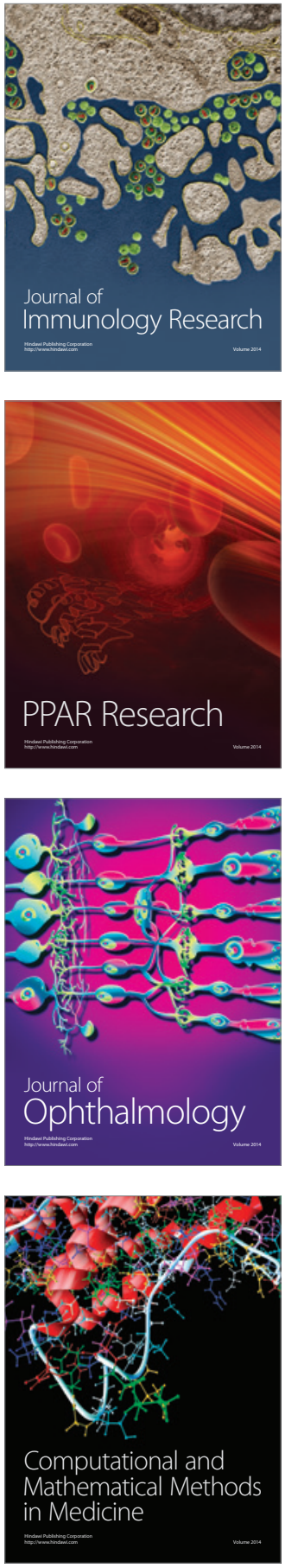

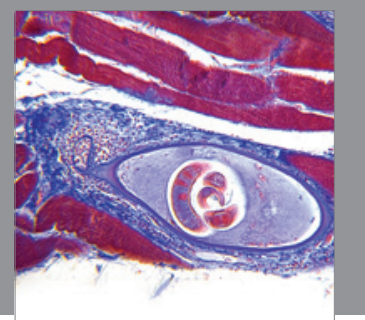

Gastroenterology

Research and Practice
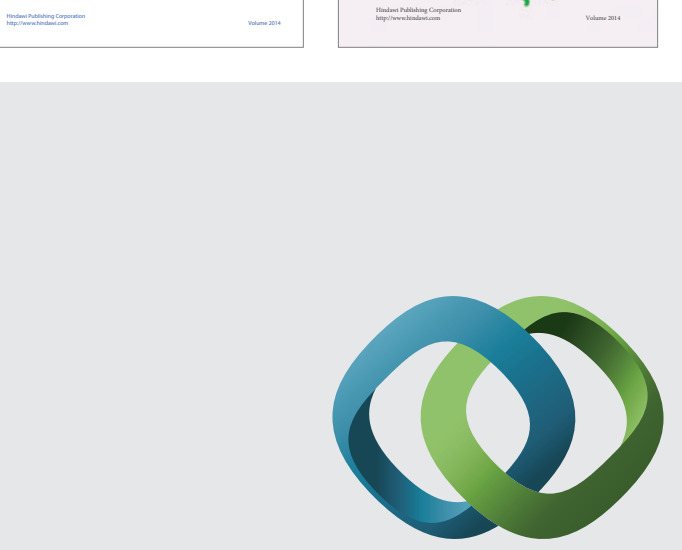

\section{Hindawi}

Submit your manuscripts at

http://www.hindawi.com
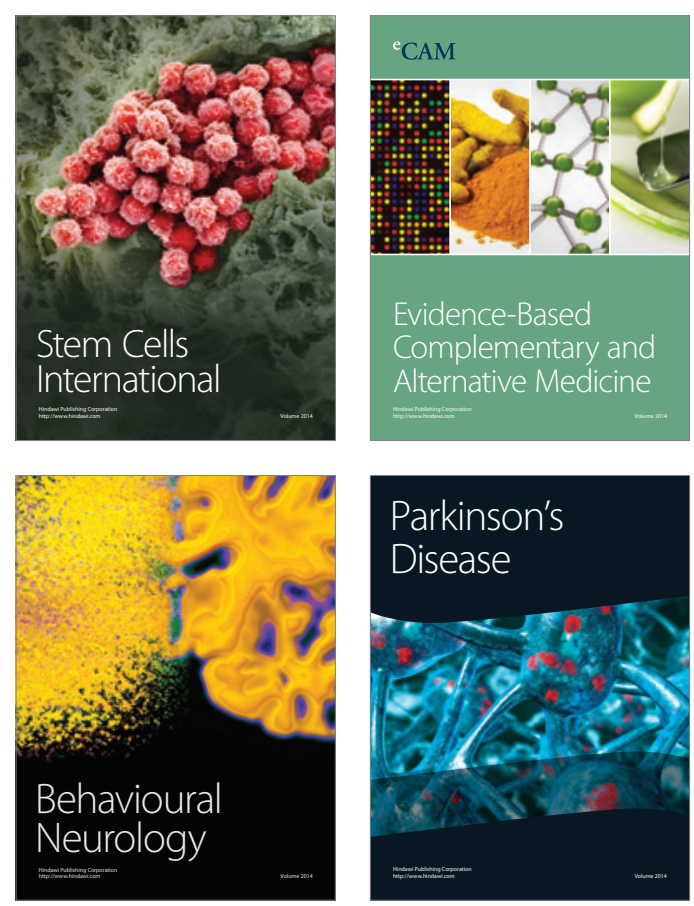

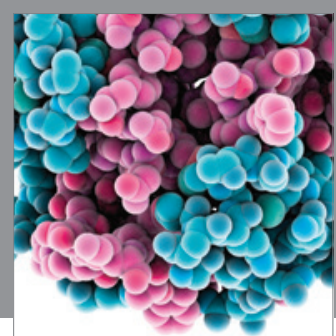

Journal of
Diabetes Research

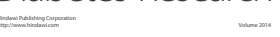

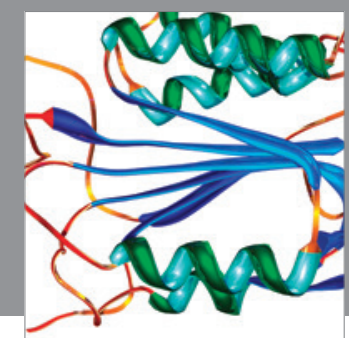

Disease Markers
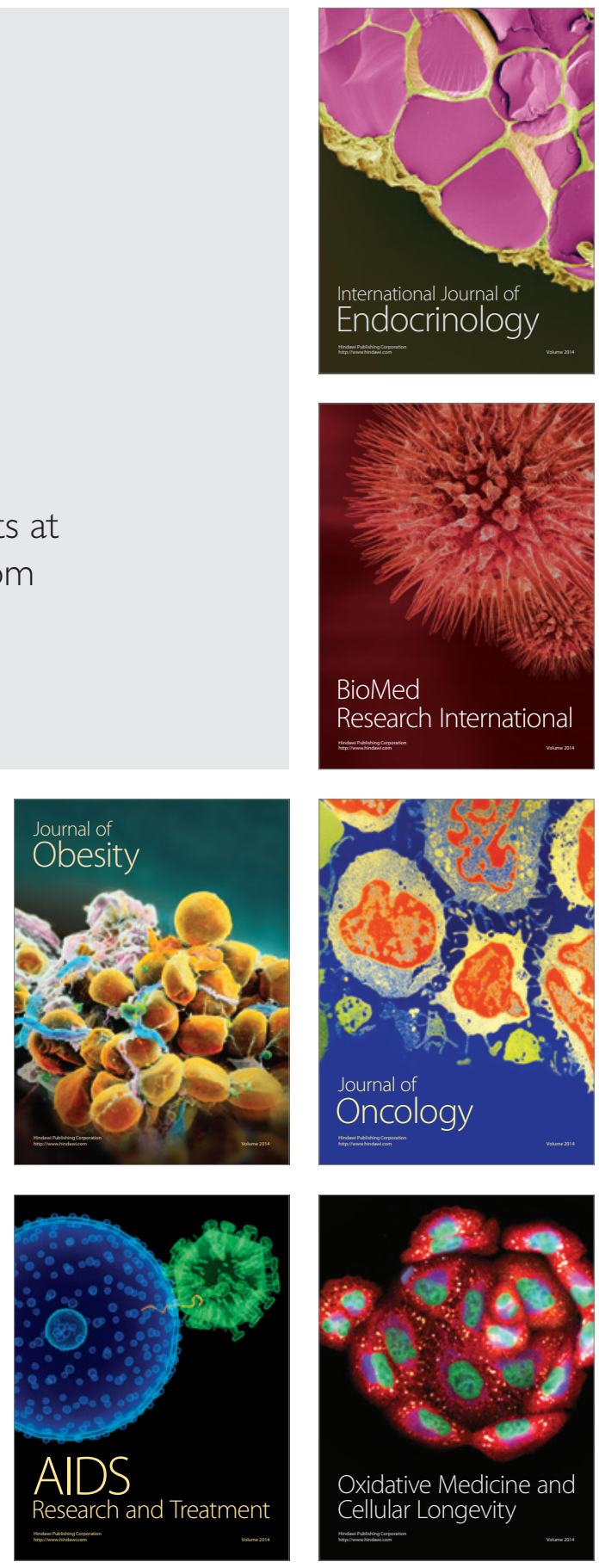